\title{
Insulin Resistance Modification is a Game Changer for Type 2 Diabetes Treatment Strategy
}

\author{
Juraj Gmitrov* \\ Krompachy Hospital, Agel SK inc., Diabetology Clinic, Slovakia
}

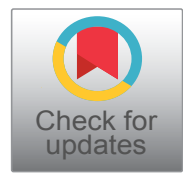

*Corresponding author: Juraj Gmitrov, Krompachy Hospital, Agel SK Inc., Diabetology Clinic, Banicka stvrt 1, Krompachy, 05642, Slovakia

\begin{abstract}
Insulin resistance, appearing years before diabetes main diagnostic sign, hyperglycemia, stands in the origin of type 2 diabetes (T2DM), generated by complex impairment of a spectrum biochemical processes the most important of which is the failure of phosphatidylinositol 3-kinase enzymatic chain responsible for glucose uptake and endothelial nitric oxide (NO) synthase activation. Therefore in conditions with insulin resistance the impairment of glucose uptake is strongly coupled with NO deficit and severe vasodilatory dysfunction. However T2DM conventional treatment even worsens diabetes fundamental pathophysiological mechanisms reflected by aggravated obesity and insulin resistance. These may generate vasodilatory dysfunction, arterial hypertension and dyslipidemia, diminishing intensive glycemic control mainly microvascular benefit. Glucocentric model of T2DM management should be replaced by approach focused primarily to impede insulin resistance rather then manage its cardiometabolic consequences. Antidiabetics should be selected to achieve hypoglycemic goals without increase of body weight and with ability to decrease insulin resistance, the key triggering factor of global vascular impairment and T2DM cardiovascular mortality.
\end{abstract}

\section{Keywords}

Sympathetic overactivity, Insulin resistance, Nitric oxide deficit, Cardiovascular risk

Taoist thinking postulates the route to be more important than goal being the goal by itself. Applying for type 2 diabetes (T2DM), the method is crucial how to achieve glycemic goals but at the same time do not amplify insulin resistance, the cornerstone of diabetes patophysiology and the key cardiovascular risk factor.

Insulin resistance, appearing years before the outbreak of hyperglycemia is generated by complex im- pairment of a spectrum biochemical processes the most important of which is the failure of phosphatidylinositol 3-kinase enzymatic chain responsible for glucose uptake, endothelial nitric oxide (NO) synthase activation and NO supplementation [1].

NO is a highly reactive signaling molecule with basic role in an array of fundamental intracellular processes leading to vasorelaxation, inhibition of platelet aggregation, suppression of vascular smooth muscle cell proliferation, cardiovascular remodeling and atherosclerosis $[2,3]$. NO is permanently secreted by endothelial cells opposing vasoconstrictor effect of tonic sympathetic drive [4], contributes to arterial baroreflex and vascular blood pressure buffering mechanisms [5], and participates in fine regulation of microcirculatory blood flow adjusted to actual metabolic demands. Therefore in insulin resistant states NO deficit is coupled with severe vasodilatory dysfunction, generating a major cardiovascular risk even before the manifestation of hyperglycemia.

The meta-analysis of 87 studies with 951,083 participants showed that individuals with metabolic syndrome but without hyperglycemia have similarly malignant cardiovascular outcomes as overt T2DM [6]. The relative risk of cardiovascular mortality in this study was: $1.75-2.40$ (insulin resistance - overt diabetes), stroke: 1.86 - 2.27, myocardial infarction: $1.62-1.99$ and all-cause mortality: $1.32-1.58$. The minor position of hyperglycemia between diabetic cardiovascular risk factors was also supported by negative results of intensive glycemic control on cardiovascular outcomes in ACCORD, ADVANCE and VADT trials [7-9], backed in addition by enhance cardiovascular mortality even 9 years after diabetes intensive

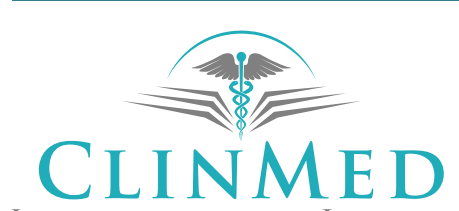

INTERNATIONAL LIBRARY 
treatment in ACCORD [10] and by absence of the legacy effect 15 years after intensive glycemic control in VADT trial [11]. Intensive glycemic control in UKPDS study was enable to prevent $80 \%$ of macrovascular and $75 \%$ microvascular complications [12] revealing a huge body of re- sidual cardiovascular risk left behind the management of hyperglycemia. Importantly, high residual cardiovascular risk still persists even after adjustment of blood pressure and hyperlipidemia [13], pointing on a powerful, underestimated cardiovascular slayer to be in play.

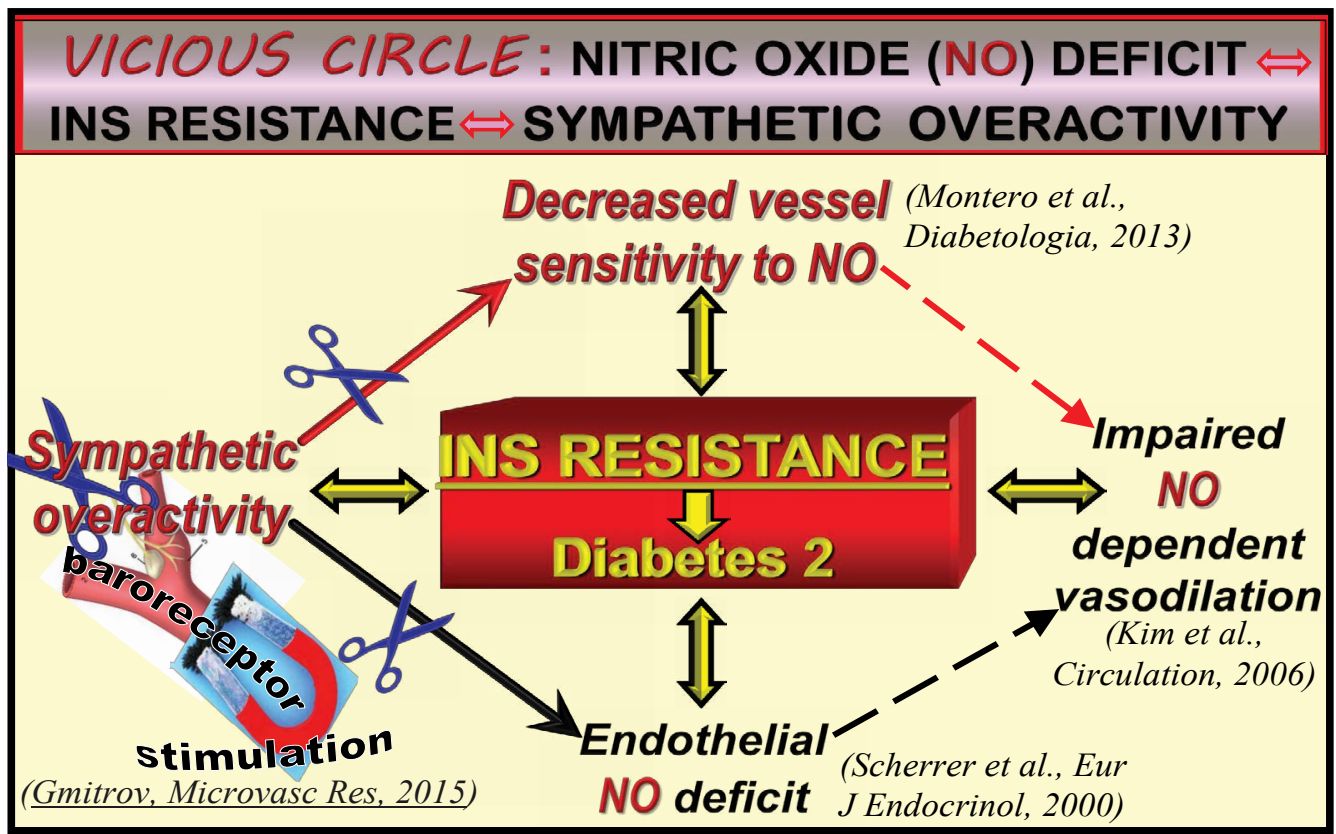

Figure 1: The vicious circle between insulin resistance, sympathetic overactivity and NO-mediated dilation, the crucial mechanism of diabetic cardiovascular impairment.

The vicious circle may be broken by augmentation of vessel sensitivity to NO- mediated dilation achieved by vascular sympathetic withdrawal, generated by carotid baroreceptor magnetic activation.

\section{INVERSE RELATIONSHIP BETWEEN MICRO- AND MACRO- VASCULAR BENEFIT OF TIGHT GLYCEMIC CONTROL}

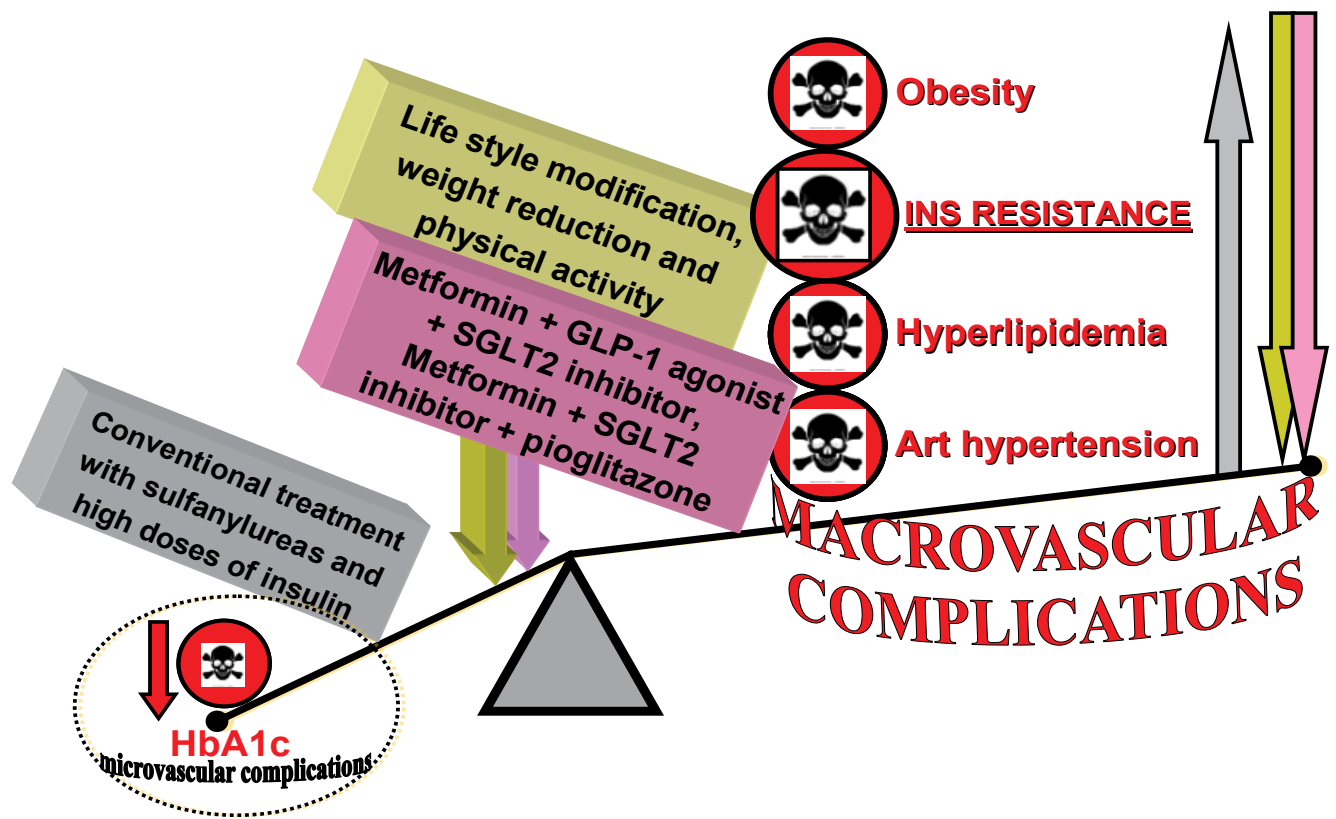

Figure 2: The lethal seesaw between micro- and macrovascular benefit of tight glycemic control with conventional drugs may be cranked by pathophysiologic approach.

Conventional glycemia depleting therapy decreases primarily microvascular complications, with reciprocal neutral or even aggravating effect on macrovacular impairment generated by worsened obesity, insulin resistance, arterial hypertension and hyperlipidemia, the "large" cardiovascular risk factors. This may result in negative net effect on cardiovascular outcomes. At the same time combination therapy with new drugs (GLP-1 agonist + SGLT2 inhibitor) generates both micro- and macrovascular protective effect with synergetic cardiovascular benefit. 
Insulin resistance, by its self propelled reciprocal relationship with endothelial NO deficit and sympathetic overactivity $[1,14]$ suggested to be the primary, durably accelerating machinery of diabetic cardiovascular structural and regulatory damage, responsible for malignant cardiovascular outcomes independently on hyperglycemia, Figure 1 . The wiring of the arterial baroreflex-mediated sympathetic withdrawal with enhanced vessel sensitivity to NO points on interaction between endothelial and autonomic vascular control mechanism [15], severely impaired in T2DM where reduced vessel sensitivity to NO-mediated dilation in addition to its chronic deficit is present [16]. However T2DM conventional treatment even worsens diabetes fundamental pathophysiological mechanisms reflected by autonomic dysfunction and aggravated obesity, a reliable visible sign of progressing insulin resistance. These promote arterial hypertension and dyslipidemia, the "large" macrovascular risk factors, diminishing thus intensive glycemic control mainly microvascular cardiovascular benefit Figure 2.

It seems that in clinical practice is not taken into consideration enough the common knowledge that T2DM is a completely different condition than type 1 diabetes and rigidly pursuing the paradigm of tight glycemic control. This approach is even boosted by deeply anchored laic judgment linking diabetes only with hyperglycemia. Intensive glycemic control is useful to prevent cardiovascular complications primarily in younger patients and may be harmful in older individuals with longer history of T2DM and pre-existing cardiovascular diseases [17]. It is crucial persuade patients to change life stile, intensify physical activity and decrease obesity. At least $10 \%$ weight reduction achieved by diet combined with aerobic and resistance exercises seems to be the most effective method to accomplish significant and sustained decrease of insulin resistance in diabetic and nondiabetic obese individuals [18].

Governments should be directly involved in T2DM prevention and treatment. State owned and private facilities (farms, camps, spas) should be established with high quality weight reduction and healthy life style modification programs. Antidiabetics should be selected to achieve hypoglycemic goals at least without weight gain, but better with capacity to decrease obesity and insulin resistance, and with evidence-based cardiovascular protective properties. Treatment should begin with dual or triple combinations, simultaneously targeting different constituents of diabetes pathophysiology, such as: metformin + pioglitazone + SGLT2 inhibitor or metformin + GLP-1 agonist + SGLT2 inhibitor, Figure 2. In particular the combination of GLP-1 agonist and SGLT2 inhibitor seems to be promising by its synergetic effect on renal and heart failure probably by vascular insulin resistance impeding - NO boosting mechanism [19], potentiated in addition by cardioprotective effect of increased myocardial tissue sensitivity to insulin [20].
This mechanisms may partially explain the property of this combination to decrease systolic blood pressure, body weight and $\mathrm{HbA1c}$, generating favorable cardiovascular outcomes [21].

Combination therapy approach fits diabetes pathophysiology, allowing to address both micro- and macrovascular complications including high residual cardiovascular risk left behind the management of the hyperglycemia generating a potential to significantly improve cardiovascular outcomes. Of course, such T2DM entrance treatment has strong economic limitations. However, favorable cardiovascular impact of each drug may generate durable, synergistic cardioprotective effect in combination, making this approach in perspective even economically advantageous.

Interestingly, a link may exist between T2DM hypotensive and hypoglycemic treatment. Hypotensives which decrease cardiovascular mortality (AT-1 receptor blockers and ACE-inhibitors) were found effectively ameliorate endothelial dysfunction, sympathetic overactivity and, importantly, insulin resistance, preventing thus new onset T2DM [22]. This suggests interrelated etiopathogenetic background of T2DM and hypertensive cardiac disease severity of which depends on the magnitude of preexisting insulin resistance [23]. The property of the arterial baroreflex-mediated vascular sympathetic withdrawal to augment vessel sensitivity to NO proposed to be the right feature to cut off the vicious circle between endothelial NO deficit, sympathetic overactivity and insulin resistance, the cornerstone of diabetic cardiovascular injury, Figure 1 [24].

And in conclusion, the glucocentric model of T2DM management should be replaced by approach focused primarily to impede insulin resistance rather then simply manage its cardiometabolic consequences, such as arterial hypertension or hyperglycemia. Hyperinsulinemic drugs (sulfonylureas or insulin) should be administered in minimal amounts to avoid obesity and insulin resistance, the key triggering factor of global vascular impairment and T2DM cardiovascular mortality.

\section{References}

1. Kim JA, Montagnani M, Koh KK, Quonet MJ (2006) Reciprocal relationships between insulin resistance and endothelial dysfunction: Molecular and pathophysiological mechanisms. Circulation 113: 1888-1904.

2. Harrison DG, Cai H (2003) Endothelial control of vasomotion and nitric oxide production. Cardiol Clin 21: 289-302.

3. DeFronzo RA (2010) Insulin resistance, lipotoxicity, type 2 diabetes and atherosclerosis: The missing links. The Claude Bernard Lecture (2009). Diabetologia 53: 1270-1287.

4. Gamboa A, Okamoto LE, Diedrich A, Choi L, Robertson D, et al. (2012) Sympathetic activation and nitric oxide function in early hypertension. Am J Physiol Heart Circ Physiol 302: 1438-1443.

5. Gmitrov J (2010) Static magnetic field blood pressure buffering, baroreflex vs. vascular blood pressure control mechanism. Int J Radiat Biol 86: 89-101. 
6. Mottillo S, Filion KB, Genest J, Joseph L, Pilote L, et al. (2010) The metabolic syndrome and cardiovascular risk a systematic review and meta-analysis. J Am Coll Cardiol 56: 1113-1132.

7. Action to Control Cardiovascular Risk in Diabetes Study Group, Gerstein HC, Miller ME, Byington RP, Goff DC Jr, et al. (2008) Effects of intensive glucose lowering in type 2 diabetes. N Engl J Med 358: 2545-2559.

8. ADVANCE Collaborative Group, Patel A, MacMahon S, Chalmers J, Neal B, et al. (2008) Intensive blood glucose control and vascular outcomes in patients with type 2 diabetes. N Engl J Med 358: 2560-2572.

9. Duckworth W, Abraira C, Mortiz T, Reda D, Emanuele N, et al. (2009) Glucose control and vascular complications in veterans with type 2 diabetes. N Engl J Med 360: 129-139.

10. ACCORD Study Group (2016) Nine-Year effects of 3.7 years of intensive glycemic control on cardiovascular outcomes. Diabetes Care 39: 701-708.

11. Wiitala WL (2018) The veterans affairs diabetes trial (VADT) at 15 years. American Diabetes Association $78^{\text {th }}$ Scientific Sessions; Orlando, Fla.

12. (1998) Intensive blood-glucose control with sulphonylureas or insulin compared with conventional treatment and risk of complications in patients with type 2 diabetes (UKPDS 33). UK Prospective Diabetes Study (UKPDS) Group. Lancet 352: 837-853.

13. Blacher J, Evans A, Arveiler D, Amouyel P, Ferrières J, et al. (2010) Residual cardiovascular risk in treated hypertension and hyperlipidaemia: The PRIME Study. J Hum Hypertens 24: 19-26.

14. Scherrer U, Sartori C (2000) Defective nitric oxide synthesis: A link between metabolic insulin resistance, sympathetic overactivity and cardiovascular morbidity. Eur J Endocrinol 142: 315-323.
15. Gmitrov J (2015) Baroreceptor stimulation enhanced nitric oxide vasodilator responsiveness, a new aspect of baroreflex physiology. Microvasc Res 98: 139-144.

16. Montero D, Walther G, Pérez-Martin A, Vicente-Salar N, Roche E, et al. (2013) Vascular smooth muscle function in type 2 diabetes mellitus: A systematic review and meta-analysis. Diabetologia 56: 2122-2133.

17. DeFronzo RA, Eldor R, Abdul-Ghani M (2013) Pathophysiologic approach to therapy in patients with newly diagnosed type 2 diabetes. Diabetes Care 36: S127-S138.

18. Bird SR, Hawley JA (2017) Update on the effects of physical activity on insulin sensitivity in humans. BMJ Open Sport Exerc Med 2: e000143.

19. Busch RS, Kane MP (2017) Combination SGLT2 inhibitor and GLP-1 receptor agonist therapy: A complementary approach to the treatment of type 2 diabetes. Postgrad Med 129: 686-697.

20. Fuentes-Antrás J, Picatoste B, Ramírez E, Egido J, Tunon $\mathrm{J}$, et al. (2015) Targeting metabolic disturbance in the diabetic heart. Cardiovasc Diabetol 14: 17.

21. Goncalves E, Bell DSH (2018) Combination treatment of SLGT2 inhibitors and GLP-1 receptor agonists: Symbiotic effects on metabolism and cardiorenal risk. Diabetes Ther 9: 919-926.

22. Sarafidis PA, McFarlane SI, Bakris GL (2007) Antihypertensive agents, insulin sensitivity, and new-onset diabetes. Curr Diab Rep 7: 191-199.

23. Ferrannini E, Buzzigoli G, Bonadonna R, Giorico MA, Oleggini $M$, et al. (1987) Insulin resistance in essential hypertension. N Engl J Med 317: 350-357.

24. Gmitrov J (2016) Baroreflex-mediated sympathetic withdrawal enhanced vessel sensitivity to NO, a cutting point of the pathophysiological crosstalk between sympathetic overactivity, insulin resistance and NO deficit. ADA $76^{\text {th }}$ scientific session. Diabetes 65: 120. 